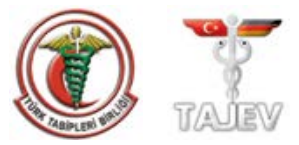

\title{
Parasitic myoma after laparoscopic surgery: a mini-review
}

\author{
Hakan Erenel, Osman Temizkan, Begüm Aydoğan Mathyk, Suat Karataş \\ Clinic of Obstetrics and Gynecology, Şişli Hamidiye Etfal Training and Research Hospital, Istanbul, Turkey
}

\begin{abstract}
The aim of this review is to summarize the cases of parasitic myomas after laparoscopic surgery. A literature search was performed using the PubMed database for the period of January 1997 to December 2014. We used the following keywords: "laparoscopic hysterectomy," "laparoscopic myomectomy," "morcellation," "parasitic fibroids," "parasitic myomas," and "leiomyomatosis." A total of 29 articles meeting the selection criteria were included in our review, describing 53 patients who underwent surgery for parasitic myomas. Parasitic myoma is a rare condition resulting from the small fibroid fragments left after morcellation and can be either asymptomatic or symptomatic. Although it is rare, patients should be informed about the risk of this condition after laparoscopic surgery. It is important for surgeons to look for small fibroid fragments during and after morcellation and make an effort to remove every piece of tissue. (J Turk Ger Gynecol Assoc 2015; 16: 181-6)

Keywords: Laparoscopic hysterectomy, laparoscopic myomectomy, morcellation, parasitic fibroids, parasitic myomas
\end{abstract}

Received: 18 January, 2015

Accepted: 29 April, 2015

Available Online Date: 14 July, 2015

Parasitic myoma is a term used to describe a myoma of extrauterine nourishing. Although uterine myomas are the most common female tumors, parasitic myomas are rare pathologic structures of uncertain etiology. One theory suggests that pedunculated subserosal myomas become separated from the uterus and receive blood supply from other adjacent organs, such as the bowel, peritoneum, omentum, or mesentery (1). Peritoneal metaplasia is another theory that describes the pathogenesis of myomas in unexpected fields of abdomen. The development of multiple nodules on peritoneal surfaces is referred to as leiomyomatosis peritonealis disseminata (LPD), which was first described in 1952 by Wilson et al. (2). Different pathological mechanisms related to hormonal factors, genetic basis, pregnancy, oral contraceptive pills, and prior surgery have been described in the literature. Estrogen exposure can stimulate metaplasia and differentiation of subperitoneal mesenchymal stem cells to smooth muscle cells (3). LPD is usually considered as a premenopausal benign condition; however, malignant transformation and postmenopausal status have also been observed in exceptional cases $(4,5)$. A recent report showed that currently, there are approximately 200 cases of LPD (6). In the last decade, there have been increasing reports of parasitic myomas after laparoscopic surgery, which have been newly classified as iatrogenic parasitic myomas (7). These myomas are related to the small fibroid fragments left after morcellation that could have detached from the uterus and developed blood supply from adjacent organs. In this paper, we aimed to summarize and discuss the various reports of parasitic myomas after laparoscopic uterine surgery.
This systematic review was conducted in accordance with the PRISMA guidelines. Literature search was performed using the PubMed database for the period of January 1997 to December 2014. We used following keywords: "laparoscopic hysterectomy," "laparoscopic myomectomy," "morcellation," "parasitic fibroids," "parasitic myomas," and "leiomyomatosis." Specifically, reports written in English language and in which patients with parasitic myoma underwent laparoscopic uterine surgery were considered eligible for our review. Articles including patients who underwent laparotomy or vaginal surgery or who were operated on account of retained myoma in the initial surgery were excluded. Reports with malignant pathology results were also excluded from our review. The flow chart for the study selection process is shown in Figure 1. From the selected articles, the number, size, receptor status, location of parasitic myomas, usage of morcellator in previous surgery, and type of previous laparoscopic uterine surgery were determined.

After the initial literature search, 36 articles were identified for review. However, after screening the language, 2 reports were excluded because they were not written in English. Of the remaining 34 reports, 5 did not meet the inclusion criteria and were thus excluded (4 reports involved previous laparotomy and 1 involved retained myoma in an initial surgery). Consequently, 29 reports were finally included in our review, describing 53 patients who underwent surgery for parasitic myomas. The average age of the patients at diagnosis was 40 years (range: $24-57$ years). Of the total patients selected, 31 (59\%) had undergone laparoscopic myomectomy, 12 (23\%)

Address for Correspondence: Hakan Erenel, Şişli Hamidiye Etfal Eğitim ve Araştırma Hastanesi, Kadın Hastalıkları ve Doğum Kliniği, İstanbul, Türkiye. 


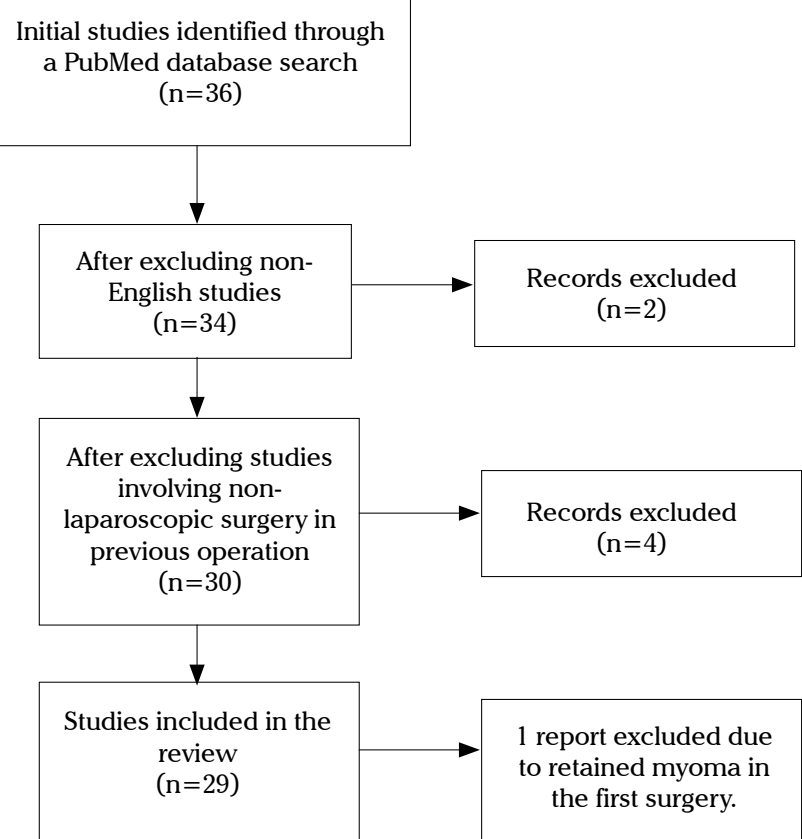

Figure 1. Flow chart of the literature search

had undergone laparoscopic subtotal hysterectomy, 6 (11\%) had undergone total laparoscopic hysterectomy, 3 (6\%) had undergone laparoscopic-assisted myomectomy, and 1 had undergone laparoscopic endometriosis surgery. In addition, 28 of the 53 patients (53\%) had complained of abdominal pain; however, 13 of 53 patients (25\%) were asymptomatic. The average time between the initial laparoscopic uterine surgery and the onset of symptoms related to parasitic myomas was 57 months (range:2-192 months). In the selected studies, parasitic myomas were most commonly found in the colon serosa and pouch of Douglas. The largest observed myoma was sized 30 $\mathrm{cm}$ and was attached to the omentum. Because we excluded malignant cases, all the cases reported in this review refer to a benign pathology. The list of all studies considered in this review is provided in Table 1.

In 1909, parasitic myomas were first described as rare pathologic structures believed to be pedunculated subserosal myomas that twist from the uterine pedicle and survive by neovascularization of adjacent organs such as the omentum and mesenteric vessels $(1,8)$. The first report describing parasitic myomas after laparoscopic surgery was published in 1997 (9). Morcellation was not performed in this case and the parasitic myoma was located near the trocar sleeve and had grown into the abdominal wall. Parasitic myomas can be found in variable locations. They are commonly reported in the pelvic region; however, Sinha et al. (10) have reported a case of parasitic myoma under the diaphragm. The degree of Trendelenburg position may be related to the location of parasitic myomas. Thus, we propose that the International Federation of Gynecology and Obstetrics (FIGO) classification system for uterine myomas and parasitic myomas should classify parasitic myomas on the basis of their locations in the mesentery, omentum, bowel wall, or peritoneum.
A retrospective study has shown a prevalence of $0.9 \%$ for parasitic myomas after laparoscopic surgery using morcellation (11). Because the symptoms of parasitic myomas are not specific, the number of case reports with asymptomatic presentation should not be underestimated (12-16). In our review, 13 of 53 patients (25\%) were asymptomatic. Small and asymptomatic parasitic myomas can be overlooked at follow-up.

Our review also consisted of reports on pelvic adenomyotic masses after laparoscopic subtotal hysterectomies in the literature $(17,18)$. Morcellation of the uterus rather than that of the myoma could be associated with adenomyotic masses. Other issues related to uterine morcellation include the dissemination of occult malignancies including uterine sarcoma and its possible negative effect on patient prognosis. Morcellation within a surgical bag can be a safe option to prevent dissemination of undetected uterine malignancies (19) and can also prevent the scattering of small myoma fragments, which is probably the most important risk factor for iatrogenic parasitic myomas.

The literature shows variable intervals between the first surgery and the diagnosis of parasitic myomas, ranging from 2 to 192 months. Circulating hormone levels and the receptor status can affect the onset of clinical symptoms and potential growth patterns. Our literature review indicates that different descriptions are used for the pathogenesis of parasitic myomas, including disseminated peritoneal leiomyomatosis, parasitic peritoneal leiomyomatosis, multiple ectopic myomas, and peritoneal leiomyoma (12, 20-22). Disseminated peritoneal leiomyomatosis is a rare spontaneous condition that should be differentiated from iatrogenic parasitic myomas (23). Thus, we recommend the establishment of a nomenclature to eliminate the pathogenic diversity of parasitic myomas in the literature.

With advances in gynecologic laparoscopic surgery, iatrogenic parasitic myomas after laparoscopic uterine surgery became a new issue. In our review, 48 of 53 patients were reported between 2007 and 2014. Most of these cases involved a history of morcellation. Morcellation of myomas during myomectomy and morcellation of the uterus during hysterectomy appear to be risk factors for iatrogenic parasitic myomas. LaCoursiere et al. (24) reported a case of retained fragments after laparoscopic total hysterectomy, which showed endocervical fragments in the cul de sac.

In our review, $25 \%$ of patients were asymptomatic, which can be related to the size and location of parasitic myomas. On the other hand, a review of the literature showed different sizes of myomas that were located on the bowel wall. Further studies should demonstrate the necessity of surgery for the treatment of asymptomatic small parasitic myomas because these myomas can attach very firmly to the bowel or mesentery. Leren et al. (25) have reported a case of intestinal perforation during surgery for parasitic myomas measuring $5 \mathrm{~cm}$. Aust et al. (26) have reported a case of parasitic myoma resembling ovarian malignancy in which surgery was performed by a gynecologic oncologist. Extensive and complicated surgery may be required for parasitic myomas. Patients should be informed about this extensive surgery. The pararectal fossa, abdominal wall trocar site, omentum, appendix, paravesical space, gastric serosa, intestinal serosa, subcutaneous tissue, lumbar region, rectus 


\begin{tabular}{|c|c|c|c|c|c|c|c|c|c|c|c|c|c|c|}
\hline 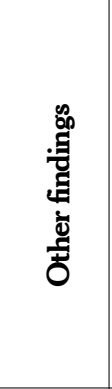 & 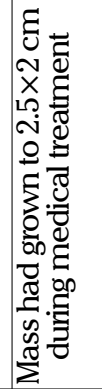 & 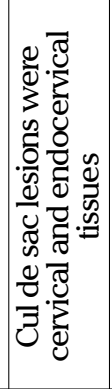 & & & & 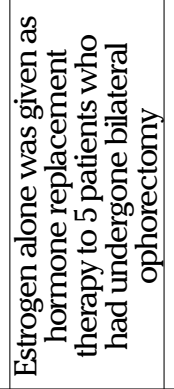 & & & 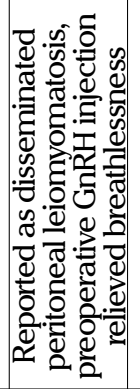 & & & & 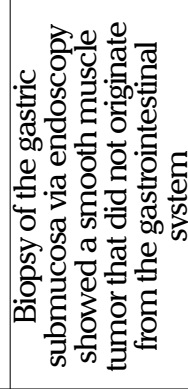 & 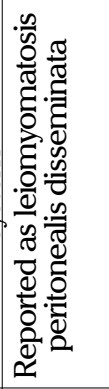 \\
\hline 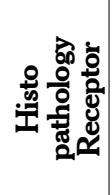 & 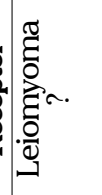 & 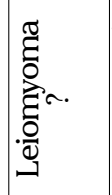 & 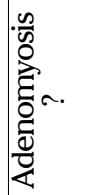 & 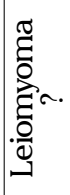 & 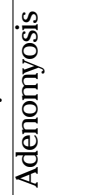 & 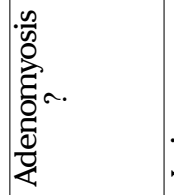 & 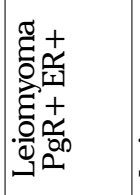 & 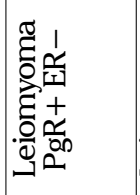 & 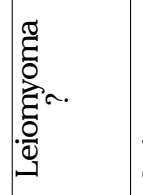 & 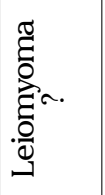 & 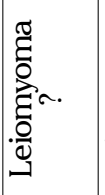 & 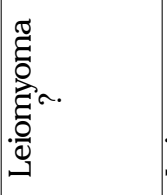 & 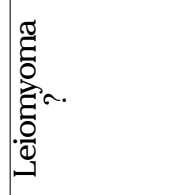 & 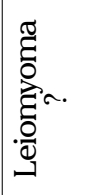 \\
\hline 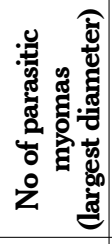 & $-\xi$ & 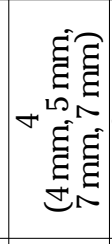 & 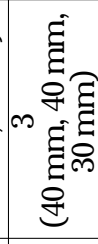 & $\infty$ & $-\underset{g}{q}$ & 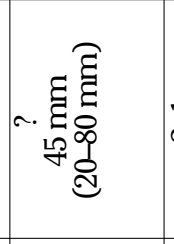 & 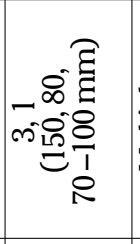 & 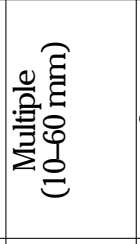 & 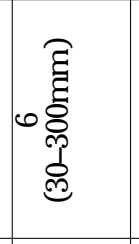 & $-\underset{\tilde{E}}{E}$ & 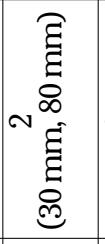 & ๙. & 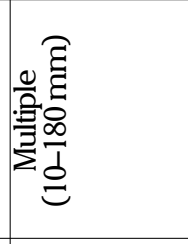 & 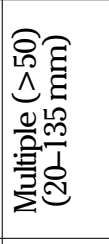 \\
\hline 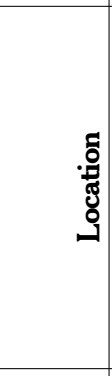 & 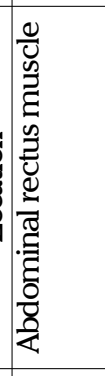 & 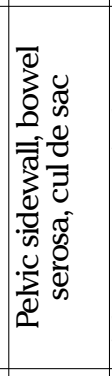 & 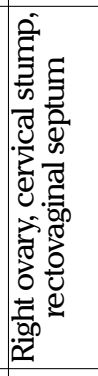 & 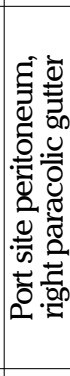 & 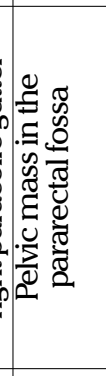 & 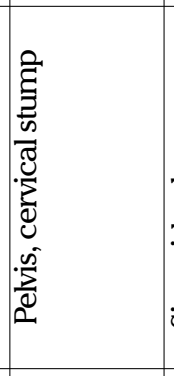 & 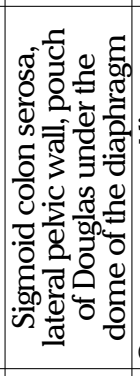 & 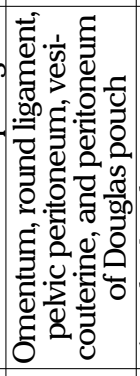 & 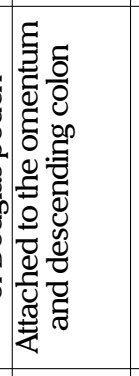 & 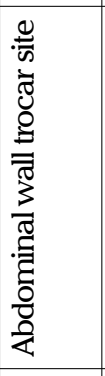 & 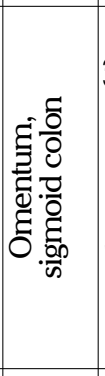 & 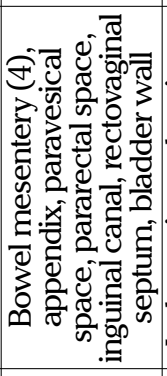 & 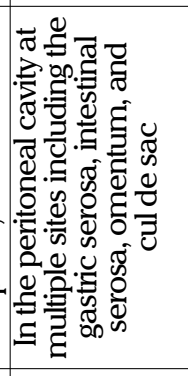 & 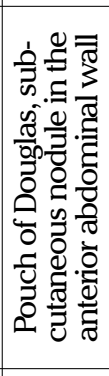 \\
\hline 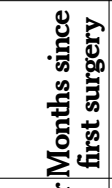 & & 은 & $\cong$ & ஓి & 8 & 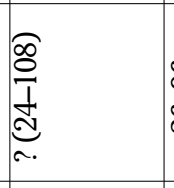 & $\begin{array}{l}\mathscr{8} \\
\dot{8}\end{array}$ & $\stackrel{N}{ }$ & os & $\mathscr{\rho}$ & $\infty$ & 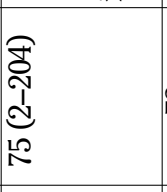 & $\mathfrak{N}$ & 尺 \\
\hline 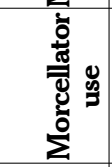 & 2 & $£$ & 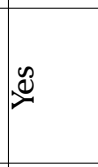 & 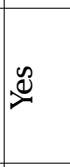 & 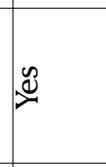 & 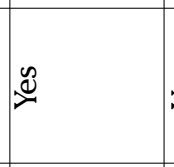 & 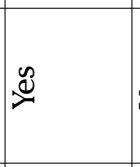 & 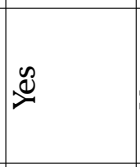 & $£$ & 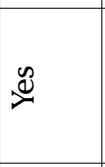 & 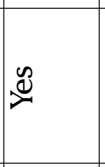 & 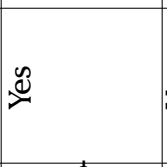 & $\stackrel{\Downarrow}{x}$ & 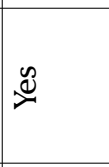 \\
\hline 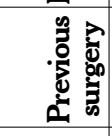 & & $\stackrel{I}{\stackrel{I}{J}}$ & 萦 & $\sum_{-1}$ & 萦 & 焉 & 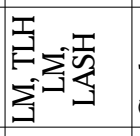 & 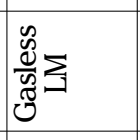 & $\sum_{-1}$ & $\sum_{-1}$ & $\sum_{3}$ & 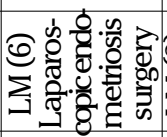 & 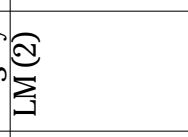 & $\sum_{3}$ \\
\hline 竞: & $\mathscr{F}$ & లి & 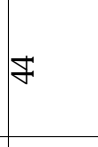 & $\stackrel{\infty}{\sim}$ & iొ & $\frac{\mathscr{O}}{d}$ & $\begin{array}{l}\stackrel{\infty}{0} \\
\stackrel{F}{F}\end{array}$ & శా & $\stackrel{\nabla}{N}$ & ले & ஜ্ & 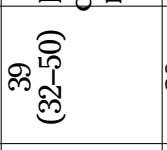 & లో & 㧒 \\
\hline 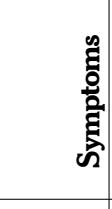 & 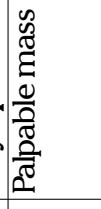 & 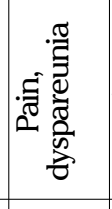 & ๘ & 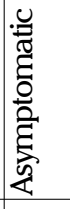 & 章 & 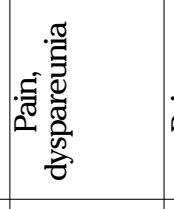 & 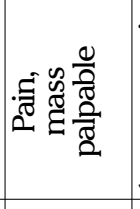 & 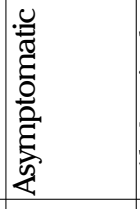 & 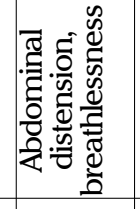 & 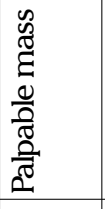 & 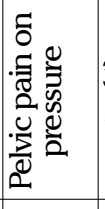 & 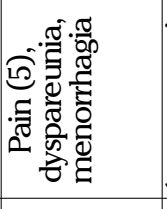 & 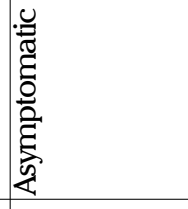 & 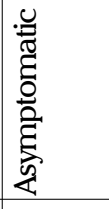 \\
\hline 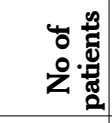 & - & - & - & - & - & $\infty$ & $\sim$ & - & - & - & - & $\sim$ & - & - \\
\hline 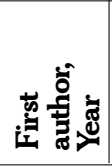 & 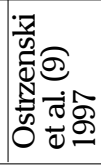 & 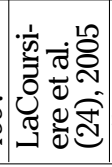 & 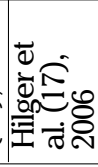 & 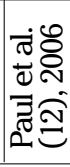 & 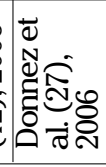 & 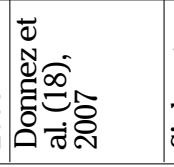 & 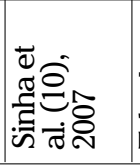 & 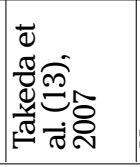 & 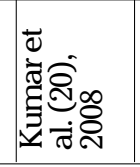 & 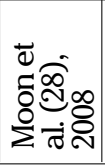 & 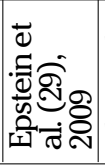 & 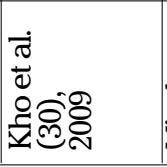 & 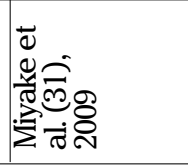 & 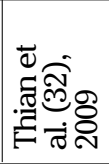 \\
\hline
\end{tabular}




\begin{tabular}{|c|c|c|c|c|c|c|c|c|c|c|c|c|c|c|c|}
\hline & 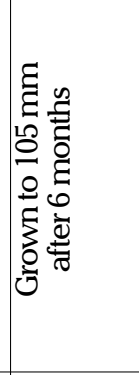 & 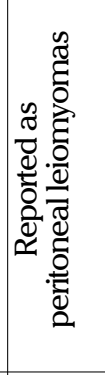 & & 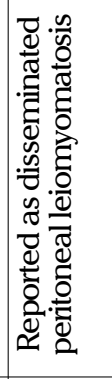 & 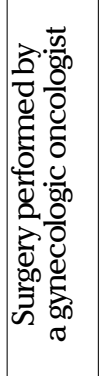 & & 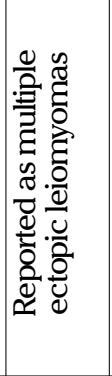 & 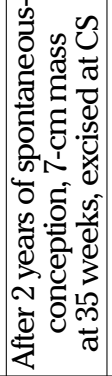 & 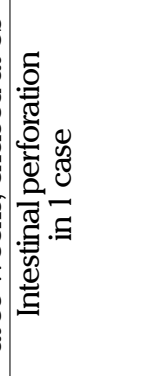 & 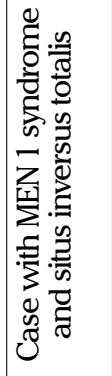 & & & & & 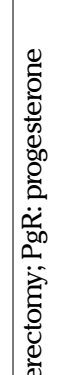 \\
\hline 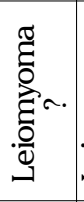 & 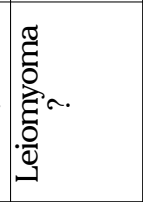 & 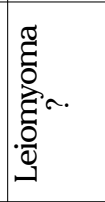 & 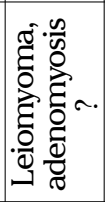 & 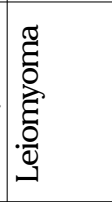 & 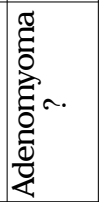 & 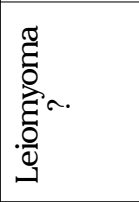 & 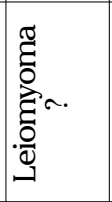 & 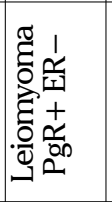 & 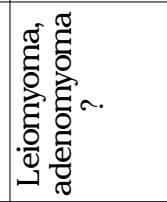 & 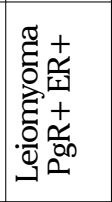 & 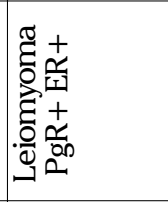 & 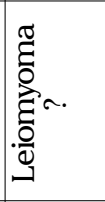 & 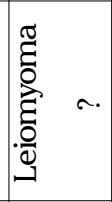 & 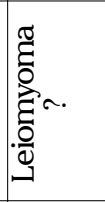 & 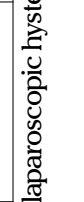 \\
\hline 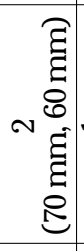 & $-\underset{\infty}{\xi}$ & 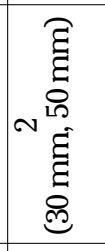 & 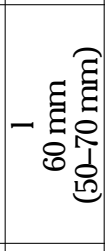 & $\begin{array}{l}\text { हี } \\
-\frac{8}{8} \\
\text { है }\end{array}$ & م & 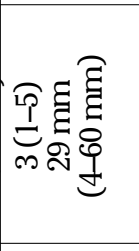 & 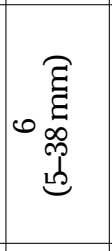 & $-\underset{\Xi}{\Xi}$ & 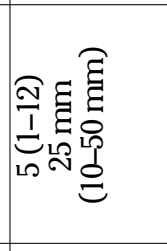 & 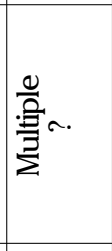 & ฉ & 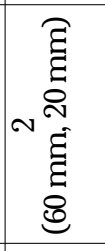 & 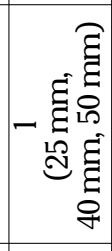 & $-\underset{\xi}{\Xi}$ & 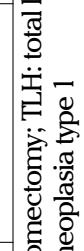 \\
\hline 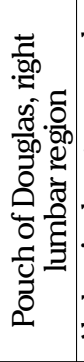 & 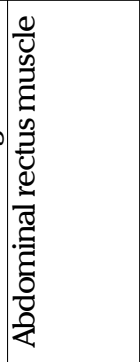 & 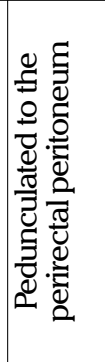 & 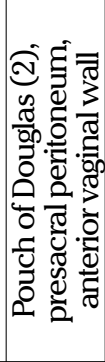 & 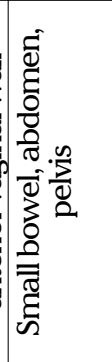 & 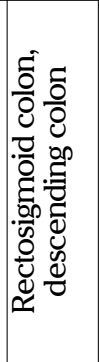 & 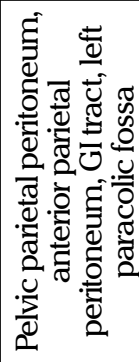 & 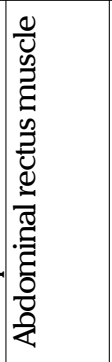 & 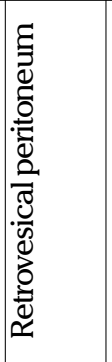 & 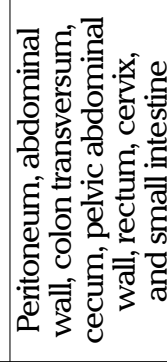 & 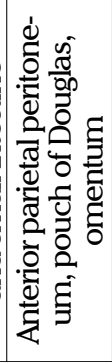 & 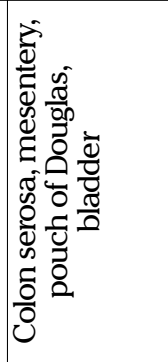 & 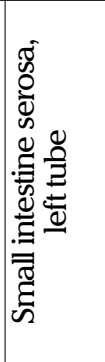 & 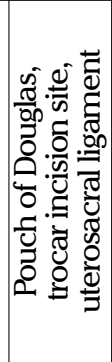 & 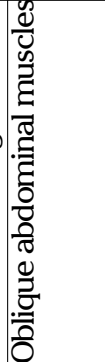 & 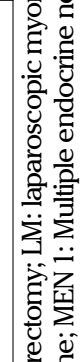 \\
\hline$\ddot{~}$ & $\stackrel{\infty}{\sim}$ & $\nexists$ & 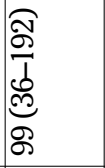 & హ & బి & 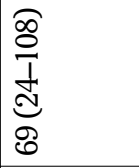 & $\stackrel{\overbrace{}}{\beth}$ & $\stackrel{\sim}{\sim}$ & 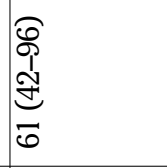 & \$ & $\stackrel{\infty}{\sim}$ & $\varpi_{\infty}$ & 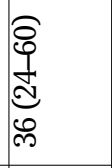 & $\infty$ & 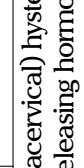 \\
\hline$\stackrel{\mathscr{Z}}{\mathscr{Z}}$ & $\stackrel{0}{2}$ & $£$ & $\mathscr{ટ}$ & $£$ & r. & 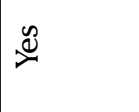 & 之 & 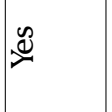 & $£$ & $£$ & 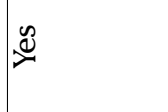 & $£$ & a. & 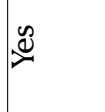 & 웡 \\
\hline$\sum_{3}$ & 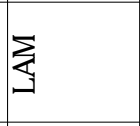 & $\sum_{1}$ & 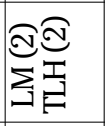 & 忈 & $\stackrel{I}{J}$ & $\sum_{3}$ & 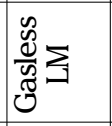 & 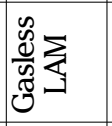 & $\sum_{j}$ & 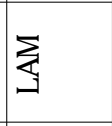 & $\sum_{I}$ & $\sum_{j}$ & 甹 & $\stackrel{\Xi}{\rightleftarrows}$ & 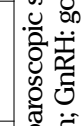 \\
\hline F & $\bar{m}$ & $\stackrel{9}{+}$ & F & $\stackrel{\infty}{+}$ & $F$ & \& & $F$ & 尺ి & 总 & $\bar{m}$ & Lి & চ্র & F & $\stackrel{\infty}{\infty}$ & 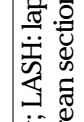 \\
\hline : & 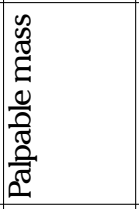 & 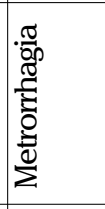 & 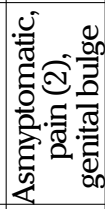 & 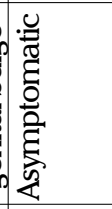 & 苂 & 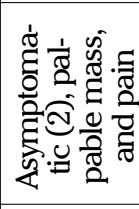 & 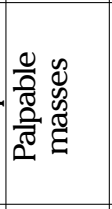 & 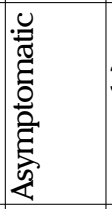 & 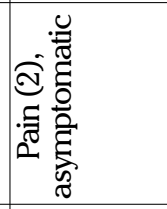 & 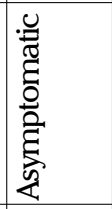 & 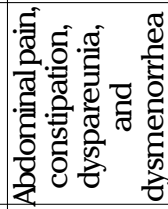 & 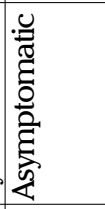 & 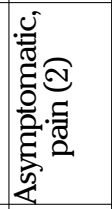 & .5 & 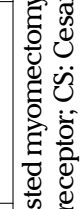 \\
\hline- & - & - & $\nabla$ & - & - & $\sigma$ & - & - & $m$ & - & - & - & $m$ & - & : \\
\hline 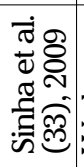 & 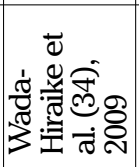 & 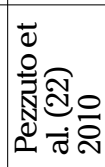 & 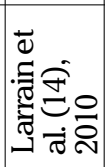 & 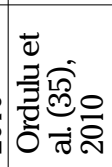 & 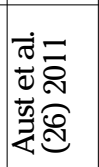 & 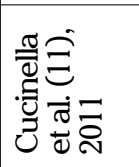 & 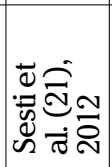 & 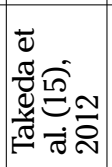 & 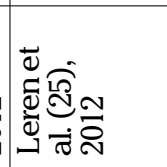 & 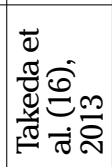 & 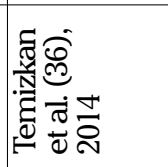 & 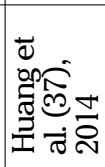 & 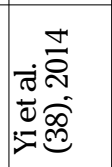 & 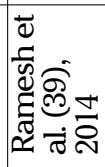 & 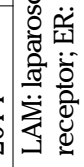 \\
\hline
\end{tabular}


muscle, bowel mesentery, and uterine tube are other locations for parasitic myomas (27-39). In the case by Kumar et al. (20) preoperative gonadotropin-releasing hormone (GnRH) injection relieved breathlessness; however, it was not effective in reducing the size of the mass significantly. Medical treatment of parasitic myomas located on the bowel wall or mesentery and the clinical course of asymptomatic small parasitic myomas should be analyzed in further studies.

The receptor status was not available in most reports. Given that the receptor status and hormone levels can affect the clinical course, further studies are needed to investigate the importance of the receptor status and hormones.

\section{Conclusion}

Fibroid remnants after laparoscopic myomectomy/hysterectomy are risk factors for the growth of fibroid tissue in the peritoneal cavity with unexpected localizations. Despite its rarity, patients should be informed about this risk. It is important for surgeons to look for small fibroid fragments during and after morcellation and accordingly make efforts to remove every piece of tissue. Furthermore, of the different types of morcellators available, it is important to select the one that provides less tissue scattering. Moreover, at the end of surgery, the pelvis should be irrigated in the reverse Trendelenburg position and surgeons should be aware of the late-onset symptoms of the disease.

\section{Peer-review: Externally peer-reviewed.}

Author Contributions: Concept - H.E., O.T., B.A.M., S.K.; Design - B.A.M., S.K.; Supervision - H.E., O.T.; Resource - H.E., B.A.M., S.K.; Materials - H.E., B.A.M., S.K.; Data Collection and/or Processing H.E., B.A.M., S.K.; Analysis and/or Interpretation - H.E., O.T.; Literature Search - H.E., B.A.M., S.K.; Writing - H.E., B.A.M.; Critical Reviews - H.E., O.T., B.A.M.

Conflict of Interest: No conflict of interest was declared by the authors.

Financial Disclosure: The authors declared that this study has received no financial support.

\section{References}

1. Brody S. Parasitic fibroid. Am J Obstet Gynecol 1953; 65: 1354-6.

2. Willson JR, Peale AR. Multiple peritoneal leiomyomas associated with a granulosa cell tumor of the ovary. Am J Obstet Gynecol 1952; 64: 204-8.

3. Tavassoli FA, Norris HJ. Peritoneal leiomyomatosis (leiomyomatosis peritonealis disseminata): a clinicopathologic study of 20 cases withultrastructural observations. Int J Gynecol Pathol 1982; 1: 59-74. [CrossRef]

4. Fulcher AS, Szucs RA. Leiomyomatosis peritonealis disseminata complicated by sarcomatous transformation and ovarian torsion:presentation of two cases and review of the literature. Abdom Imaging 1998; 23: 640-4. [CrossRef]

5. Sharma P, Chaturvedi KU, Gupta R, Nigam S. Leiomyomatosis peritonealisdisseminata with malignant change in a post-menopausal woman. Gynecol Oncol 2004; 95: 742-5. [CrossRef]

6. Yang R, Xu T, Fu Y, Cui S, Yang S, Cui M. Leiomyomatosis peritonealis disseminata associated with endometriosis: A case report and review of the literature. Oncol Lett 2015; 9: 717-20.

7. Nezhat C, Kho K. Iatrogenic myomas: new class of myomas? J Minim Invasive Gynecol 2010; 17: 544-50. [CrossRef]
8. Kelly HA, Cullen TS. Myomata of the uterus. Philadelphia: WB Saunders; 1909.

9. Ostrzenski A. Uterine leiomyoma particle growing in an abdominal-wall incision after laparoscopic retrieval. Obstet Gynecol 1997; 89: 853-4. [CrossRef]

10. Sinha R, Sundaram M, Mahajan C, Sambhus A. Multiple leiomyomas after laparoscopic hysterectomy: report of two cases. J Minim Invasive Gynecol 2007; 14: 123-7. [CrossRef]

11. Cucinella G, Granese R, Calagna G, Somigliana E, Perino A. Parasitic myomas after laparoscopic surgery: an emerging complication in the use of morcellator? Description of four cases. Fertil Steril 2011; 96: e90-6. [CrossRef]

12. Paul PG, Koshy AK. Multiple peritoneal parasitic myomas after laparoscopic myomectomy and morcellation. Fertil Steril 2006; 85: 492-3. [CrossRef]

13. Takeda A, Mori M, Sakai K, Mitsui T, Nakamura H. Parasitic peritoneal leiomyomatosis diagnosed 6 years after laparoscopic myomectomy with electric tissue morcellation: report of a case and review of the literature. J Minim Invasive Gynecol 2007; 14: 770-5. [CrossRef]

14. Larrain D, Rabischong B, Khoo CK, Botchorishvili R, Canis M, Mage G. "Iatrogenic" parasitic myomas: unusual late complication of laparoscopic morcellation procedures. J Minim Invasive Gynecol 2010; 17: 719-24. [CrossRef]

15. Takeda A, Imoto S, Mori M, Yamada J, Nakamura H. Rapid growth of parasitic myoma in early pregnancy: previously undescribed manifestation of a rare disorder after laparoscopic-assisted myomectomy. Eur J Obstet Gynecol Reprod Biol 2012; 162: 117-8. [CrossRef]

16. Takeda A, Sakurai A, Imoto S, Nakamura H. Parasitic myomas after laparoscopic-assisted myomectomy in multiple endocrine neoplasia type 1. J Obstet Gynaecol Res 2013; 39: 1098-102. [CrossRef]

17. Hilger WS, Magrina JF. Removal of pelvic leiomyomata and endometriosis five years after supracervical hysterectomy. Obstet Gynecol 2006; 108: 772-4. [CrossRef]

18. Donnez O, Squifflet J, Leconte I, Jadoul P, Donnez J. Posthysterectomy pelvic adenomyotic masses observed in 8 cases out of a series of 1405 laparoscopic subtotal hysterectomies. J Minim Invasive Gynecol 2007; 14: 156-60. [CrossRef]

19. Kho KA, Nezhat C. Evaluating the risks of electric uterine morcellation. JAMA 2014; 311: 905-6. [CrossRef]

20. Kumar S, Sharma JB, Verma D, Gupta P, Roy KK, Malhotra N. Disseminated peritoneal leiomyomatosis: an unusual complication of laparoscopic myomectomy. Arch Gynecol Obstet 2008; 278: 93-5. [CrossRef]

21. Sesti F, Di Pietro C, Capece A, Piccione E. Multiple ectopic leiomyomas of the abdominal rectus muscles after gasless laparoscopic uterine myomectomy. Arch Gynecol Obstet 2012; 285: 883-5. [CrossRef]

22. Pezzuto A, Pontrelli G, Ceccaroni M, Ferrari B, Nardelli GB, Minelli L. Case report of asymptomatic peritoneal leiomyomas. Eur J Obstet Gynecol Reprod Biol 2010; 148: 205-6. [CrossRef]

23. Advincula AP, Hernandez JC, Lieberman R. Images in reproductive medicine. Disseminated leiomyomatosis peritonei. Fertil Steril 2005; 84: 1505-7. [CrossRef]

24. LaCoursiere DY, Kennedy J, Hoffman CP. Retained fragments after total laparoscopic hysterectomy. J Minim Invasive Gynecol 2005; 12: 67-9. [CrossRef]

25. Leren V, Langebrekke A, Qvigstad E. Parasitic leiomyomas after laparoscopic surgery with morcellation. Acta Obstet Gynecol Scand 2012; 91: 1233-6. [CrossRef]

26. Aust T, Gale P, Cario G, Robertson G. Bowel resection for iatrogenic parasitic fibroids with preoperative investigations suggestive of malignancy. Fertil Steril 2011; 96: e1-3. [CrossRef] 
27. Donnez O, Jadoul P, Squifflet J, Donnez J. Iatrogenic peritoneal adenomyoma after laparoscopic subtotal hysterectomy and uterine morcellation. Fertil Steril 2006; 86: 1511-2. [CrossRef]

28. Moon HS, Koo JS, Park SH, Park GS, Choi JG, Kim SG. Parasitic leiomyoma in the abdominal wall after laparoscopic myomectomy. Fertil Steril 2008; 90: 1201.e1-2. [CrossRef]

29. Epstein JH, Nejat EJ, Tsai T. Parasitic myomas after laparoscopic myomectomy: case report. Fertil Steril 2009; 91: 932.e13-4. [CrossRef]

30. Kho KA, Nezhat C. Parasitic myomas. Obstet Gynecol 2009; 114: 611-5. [CrossRef]

31. Miyake T, Enomoto T, Ueda Y, Ikuma K, Morii E, Matsuzaki S, Murata Y. A case of disseminated peritoneal leiomyomatosis developing after laparoscope-assisted myomectomy. Gynecol Obstet Invest 2009; 67: 96-102. [CrossRef]

32. Thian YL, Tan KH, Kwek JW, Wang J, Chern B, Yam KL. Leiomyomatosis peritonealis disseminata and subcutaneous myoma--a rare complication of laparoscopic myomectomy. Abdom Imaging 2009; 34: 235-8. [CrossRef]

33. Sinha R, Sundaram M, Lakhotia S, Kadam P, Rao G, Mahajan C. Parasitic myoma after morcellation. J Gynecol Endosc Surg 2009; 1: 113-5. [CrossRef]
34. Wada-Hiraike O, Yamamoto N, Osuga Y, Yano T, Kozuma S, Taketani Y. Aberrant implantation and growth of uterine leiomyoma in the abdominal wall after laparoscopically assisted myomectomy. Fertil Steril 2009; 92: 1747.e13-5.

35. Ordulu Z, Dal Cin P, Chong WW, Choy KW, Lee C, Muto MG, et al. Disseminated peritoneal leiomyomatosis after laparoscopic supracervical hysterectomy with characteristic molecular cytogenetic findings of uterine leiomyoma. Genes Chromosomes Cancer 2010; 49: 1152-60. [CrossRef]

36. Temizkan O, Erenel H, Arici B, Asicioglu O. A case of parasitic myoma 4 years after laparoscopic myomectomy. J Minim Access Surg 2014; 10: 202-3. [CrossRef]

37. Huang PS, Chang WC, Huang SC. Iatrogenic parasitic myoma: a case report and review of the literature. Taiwan J Obstet Gynecol 2014; 53: 392-6. [CrossRef]

38. Yi C, Li L, Wang X, Liu X. Recurrence of uterine tissue residues after laparoscopic hysterectomy or myomectomy. Pak J Med Sci 2014; 30: 1134-6.

39. Ramesh B, Sharma P, Gunge D. Abdominal wall parasitic myoma following electromechanical morcellation. J Obstet Gynaecol India 2014; 64: 73-5. [CrossRef] 